\title{
A Post-colonial Analysis of Agile Software Development Methods in ICT4D
}

\author{
Scarlet Rahy10000-0002-6579-6818 · David \\ Kreps10000-0002-5776-2888 • Julian \\ M. Bass10000-0002-0570-7086 - Tarek \\ Gaber10000-0003-4065-4191 • Abdulhamid \\ Ardo10000-0002-0829-570X
}

the date of receipt and acceptance should be inserted later

\begin{abstract}
There is evidence that agile approaches to information system development can improve product quality and developer productivity. However, successful adoption of these approaches appears to depend on adaptation to specific contexts. This research contributes to a broader goal to understand what it means to "be agile" in the presence of adaptations to the specific context.

To pursue our research objectives, we have performed 31 semi-structured recorded and transcribed practitioner interviews from three companies in Lebanon. The interview transcripts were analysed using an approach informed by grounded theory.

Agile methods enable learning and improvement through team conversations. Yet, the practitioners in our study shun public self-evaluation, finding it difficult to discuss areas for improvement in public. We also found legacy "top down" management practices that undermine team autonomy and local client companies lack experience of engaging with agile processes. In a more positive vein, we found evidence of rich use of various communications channels to overcome geographical distance.

On the one hand, agile methods represent a "northern" idea being propagated to the Global South. And yet, on the other hand, the agile concept of self-organising teams has the potential to be empowering and emancipatory. Post-colonial theory helps us understand the phenomenon of agile tailoring, where development process ceremonies are adapted to suite a specific local context.
\end{abstract}

Keywords Southern Theory · Post-colonial Theory · Information Systems Development · Agile Methods · Agile Method Tailoring.

\section{Introduction}

Southern and post-colonial theories seek to look at the world from a non-Euro or US perspective. This leads to either the explicit celebration of indigenous theories, or the critique of euro-centric notions from a Southern perspective. Our aim to is

University of Salford, Greater Manchester, UK E-mail: S.Rahy@edu.salford.ac.uk http://www.salford.ac.uk 
to apply post-colonial theory to a challenge in information systems development in ICT4D in order to investigate what lessons can be learned.

Agile methods are an emerging approach to conducting ICT4D implementations that rely on iterative and incremental project deployment. Agile methods encourage self-organising teams in which developers acquire responsibility for several aspects of development projects normally assigned to project managers.

Our hypothesis, derived from previous research is that "agile methods can enhance software product quality and developer productivity in ICT4D." However, this is only possible when successfully adopted and yet adapted to the specific context.

The research questions we have identified for this study, are:

1. What can a critique, using post-colonial perspective, reveal about information system development methods?

2. What challenges do we face in using a post-colonial perspective? And,

3. What can a post-colonial perspective reveal about adaptation to a specific context of information system development methods?

In order to further our research objectives, we have employed an illustrative case study on the adoption of agile software methods in Lebanese small and medium sized companies. We conducted 31 semi-structured, open-ended, recorded and transcribed practitioner interviews, in three companies from four locations in Lebanon. The interview transcripts were analysed using a approach informed by grounded theory.

The rest of the paper is structured as follows. First we present a review of related research in agile software development. Next, we introduce our theoretical perspective based on post-colonial theory. We then discuss our research methods, focusing on research sites, data collection and data analysis. Next, we present our findings from practitioner interviews in Lebanon. We then interpret our findings in a discussion section. Finally we present conclusions, recommendations and references.

\section{Agile Methods in Information Systems Development}

Agile methods are a family of information system development methods that comprise iterative and incremental approaches [10]. Iterative development methods are cyclic, usually comprised of fixed-length time-boxes, during which a cycle of activities is performed by a software development collective. Incremental development build system functionality in a series of end-to-end functional component.

Agile methods are best described in terms of roles, ceremonies and artefacts. The roles in scrum comprise self-organising teams [16], product owners [1] and scrum masters [21]. Ceremonies are the practices and activities performed by stakeholders when performing agile methods. In scrum, ceremonies include sprint kickoff, daily stand-up meetings [29], customer demonstrations and spring retrospectives. These practices enable the orchestration of the agile software development process. Artefacts are the entities created by stakeholders during the development process (broadly defined) [2]. 


\subsection{Tailoring Agile Methods}

Agile software development methods, such as scrum and Extreme Programming (XP), were introduced under the assumption that they could be used in developing any type of project [6]. However, there are many failures in developing software using agile methods [5]. Responding to this problem, the idea of tailoring agile methods emerged where agile methods can be adapted to fit a development context such as culture or organization needs [5].

In the context of software process, software tailoring method is defined as "the adaptation of the method to the aspects, culture, objectives, environment and reality of the organisation adopting it" [5]. Such adaptation is practical as almost all software project development is unique [8]. This means deciding a software development method depends on many factors: technical, organisational or human as well as the project nature. Consequently, it is difficult to find a software development method that is most effectively adopted as it was originally suggested in textbook format [8]. Software organisations could further tailor agile methods in order to fit their country culture, values and strategies [20] [23].

To tailor an agile method, there are two main approaches: Method Engineering and Contingency Factors [5]. The Method Engineering approach makes use of meta-method processes to create a new method by using existing method fragments. The new method is developed following all software development activities related to building a software. On the other hand, tailoring software methods using the Contingency Factors approach is handled by selecting multiple existed methods and making them available to the organisation. Thus, during the development process, the method selection is achieved based on factors such as uncertainty level, impact and structure of the project being developed [5].

\subsection{Agile Methods in ICT4D}

Studies have investigated agile methods adoption in Kenya [15], Ethiopia [23] and Egypt [20]. Whereas, in Kenya where an offshore/onshore collaboration model was employed, in Ethiopia and Egypt, practitioners were adopting agile methods with little prior first hand experience. The stakeholders, in Egypt and Ethiopia, thought there might be some benefit to gained from agile methods because of reports in the grey literature and practitioner community word-of-mouth knowledge transfer.

\section{Theoretical Model}

There has been a surprising paucity of theory building underlying ICT4D [25]. Dominant research approaches have included interpretative stances [30]. Analysis in cultural studies identified how Europe came to view the rest of the world through the prism of colonialism and empire [24]. Orientalism makes a striking contrast, in terms of attitudes and actions, towards "east" and "west."

More recently, Orientalism has informed a post-colonial branch of critical management theory [14]. The study of imposition of management practices, productivity models and processes has been seen as a form of Americanization [12]. The 
binary "east/west" dichotomy has splintered into a rich and hybrid set of realities [13].

A colonial lens has been used to understand the relationship between multinational companies and their subsidiaries [19]. This ethnographic study observed the appropriation of technical knowledge and innovation which flowed from the subsidiary toward the multi-national company's overseas headquarters. Indigenous innovation conducted in an off-shore resource constrained setting has been appropriated into the private ownership of an onshore corporation.

Post-colonial theory has been used to examine the phenomenon of off-shoring in the digital economy [22]. Three main schools of thought can be identified [22]. Firstly, Orientalism [24], which rests on Foucault's [11] discourse analysis, and an appreciation of the asymmetric power relations between post-colonial countries and their former colonial masters. Secondly, a school of thought around the 'subaltern' notion developed most notably by Gayathri Spivak [27] [28]. 'Subaltern studies' focus on the 'voiceless' in global society, and the technologies of rule that maintain structures of poverty [26]. Thirdly, a school of thought that argues that the asymmetries are not "one-dimensional and one-sided as suggested by Said [but] inherently complex" [22]. This approach focusses on the complex hybrids that have developed out of the colonial era, producing syncretic mixes of practices from the different contexts of the colonised and the colonisers, and those who have been born and made their own lives and their own compromises in the complex hybrid environments that have resulted [4].

Beyond these three strands, Southern theory is a more recent contribution than post-colonial theory, that focusses on the possibilities of critical thinking taking place in, of, and about the Global South. In this view, those living in the Global South are all too often intellectually subalternised by the universal character of theoretical understandings originating in the Global North [18] [9] [7]. The rediscovered pre-colonial theoretical traditions of indigenous peoples, alongside the newly Global Southern gaze of the later descendants of the colonisers, may look back upon the Global North with a critical eye entirely of its own tenor.

This Southern Theory approach, we argue, when considered in tandem with the hybridity of Bhabha, suggests that the complex mixes of pre-colonial tradition, colonial imposition, and post-colonial adaptation, may produce unique customisations of otherwise Globally Northern impositions, and that this process may in fact be requisite both for successful adoption and for effective integration of new technologies and practices in various parts of the world.

\section{Research Methods}

This research employs a qualitative case study, with a unit of analysis comprise a set of software development practitioners employed in three Lebanese companies.

\subsection{Research Sites}

There is a paucity of large software development companies in Lebanon. Thus, we chose to sample research sites from small and medium-sized software development 
companies. Three research sites were chosen from a range of companies that implemented agile methodology in Lebanon. To protect their anonymity, the three sites were labelled LEB1, LEB2, and LEB3. LEB1 is a Lebanese based software development company having three branches in Lebanon. Its main headquarters is in Mount Lebanon and the other two branches are located in North Lebanon and Bekaa Valley. LEB1 provides solutions in areas of insurance, academics, technology, analytics, and banking for clients in Lebanon and abroad. LEB2 is software development company located in Beirut. It is specialized in developing technological solutions and digital transformation for its local and international clients. LEB2 also provides customized software consulting services. LEB3 is a software development company located in Mount Lebanon. LEB3 develops software, applications, and websites for its clients.

\subsection{Data Collection}

In-depth research interviews were conducted with 31 practitioners. The semistructured interviews were conducted in English and lasted around 40-50 minutes each. The interviews were recorded and transcribed. The interview respondent job titles and affiliations are available online [3]. The interviews were conducted faceto-face, apart from five which were conducted using Skype due to an outbreak of civil unrest on appointed date.

A semi-structured interview guide was used. Open-ended questions were used to seek out indigenous topics from respondents. Probing questions were used to encourage depth in the responses.

\subsection{Data Analysis}

All the interview transcripts were analysis using an approach informed by grounded theory. the transcripts were uploaded to the NVIVO (v11) qualitative data analysis tool [17]. The transcript data was first coded. Topics evident in the data, on a sentence-by-sentence basis, were identified. Then the codes, from different interview subjects were compared. This constant comparison technique was used to contrast the code across the respondent population. Memo writing was then used to collect the different perspective on codes from different respondents. Thus, the memos were used to represent higher-level categories identified within the data.

\section{Findings}

The following section presents the analysis of data collected from our case study. The findings discuss a critique of agile implementation in a post-colonial perspective. The findings reveal four main points: rejection of retrospectives, effective communication between teams, limitation of self-organizing teams by hierarchy, and Complications faced when dealing with Lebanese clients. 


\subsection{Teams Rejecting Retrospectives}

Practitioners find it difficult to self-evaluate their performance in front of the entire team. Practitioners fear public self-evaluation and undermine its effectiveness. Retrospectives are defined as meetings held at the end of each sprint iteration during which, team members reflect on the whole sprint and their performance. Consequently, practitioners indicated that they do not perform retrospectives which is a vital ceremony in agile. S1 explained: "We do not do retrospectives; we don't have formal evaluations." Practitioners fear public self-evaluation may cause loss of their job, hinder any promotions and undermine their position in front of colleagues. For instance, S3 explained: "If I admit my mistake in front of the whole team, I am afraid I might lose my job." Thus, practitioners would rather talk directly to the team manager and try to solve the issue covertly. A2 explained: "The problem is that there is always people who resisted talking because they were afraid that colleagues will undermine them." In addition, practitioners undermine the effectiveness of self-evaluation. Practitioners expressed how they know their responsibilities and the need to complete them. A6 expressed: "I feel everyone is self-aware. Thus, I find it useless to do retrospectives." In addition, there are misconceptions about retrospectives and their purpose. Practitioners lacked a clear understanding of what retrospectives are and their team learning purpose. Practitioners excluded retrospectives from analysing the whole team's effectiveness, celebration of its successes, and acknowledging openings for improvement. Instead of labelling a whole session for team performance evaluation, team leaders tend to encourage team members to perform this evaluation during bi-weekly meetings. V3 explained: "During our bi-weekly meetings we open indirectly the floor to learn from our mistakes. We learn from each other so that we would face the same things." Thus, team leaders would ask evaluation questions without labelling the whole session as a way to encourage members to assess their performance.

\subsection{Communication Status}

Communication is highly encouraged at the agile implementing software development companies. Diverse communication channels are open for employees. Weekly face-to-face meetings are held specifically for each project and reports are shared with all stakeholders. At the end of the week, the project manager sends a report indicating project progress to all involved teams. V4 highlighted the importance of delivering the message regardless of the technology used: "The target is that they deliver the message not the medium."

Initially, all information was supposed to be communicated through e-mail to all members involved. V3 explained: "Initially everyone was supposed to communicate in a written manner and involve everyone." This was deemed necessary in order to deliver the message and respect the institutional hierarchy. Gradually, practitioners started communicating through messaging platforms and then orally. They were training to communicate everything and involve everyone which lead to a smooth and practical communication in the development projects.

Face-to-face communication is always present among members who are in the same geographical location. When practitioners need to communicate and are present in different geographical locations, they use online video conference plat- 
forms. Communication across the diverse geographical location in Lebanon is very smooth. Our definition of geographically co-located teams differs from the conventional definition. Our co-located teams are distributed in the same country but are faced with challenges such as different culture, tradition, and religion which highly affects the person's character in Lebanon. Members do not feel the need to physically visit the different branches instead they will join virtual meetings.

The usage of communication channels depends on the needs and message being delivered. Most practitioners rely on non-written communication; as V8 expressed: "Verbal is the most common way of communicating." Especially if it is a simple update. Instant messaging platforms are also used. V4 explained: "Sometime, I have to send just an informal message so I use WhatsApp." Teams also hold meetings to update all stakeholders and send emails and reports to everyone involved. This communication strategy enables team members to communicate effectively and thoroughly.

\subsection{Restrictions of Self-Organizing teams by the hierarchy}

Hierarchy is embedded in the institutional arrangement and individual organization in software development companies. The roles assigned to practitioners, such as project manager and director, reveal that hierarchical structure is entrenched in the institutions. This leads to high levels of bureaucracy which may hinder the development process. V17 claimed: "We find that we are in a bureaucracy box; It consumes a lot of time to get one thing approved."

The hierarchy affects the teams and restricts teams from becoming self-organizing agile teams. Allocation of tasks by managers, time estimations by product owners, and micromanaging of upper management limit the development of self-organizing teams. First, managers and team leaders are responsible to assign the tasks to the team members. V7 explained: "We assign the global tasks for each team and then each team leader will assign the tasks to his/her team." This creates occasional tension between team leaders and project managers from one side, and team members from the other. Team members prefer to choose their tasks. As A7 describes: "I dislike the idea that our leaders allocate the tasks." S6 added: "It would motivation if I chose my open tasks from each sprint."

Second, the time estimation for each project is performed from all the involved departments. The first high level estimation is done by scanning the requirements. Understanding the task consumes time and requires effort prior to implementation. Time estimation is performed by product owner and team leaders which may lead to inaccurate evaluation of the time required for task completion.

Third, dealing with the pressures of the upper management is one of the main challenges faced by employees. Practitioners revealed how the CEO micro-managed the process. A7 explained: "The CEO micro-managed and interfered in every single detail." The CEO wanted to know the details of every project in the daily morning meetings. These meeting will last longer than advised and team leaders weren't able to conduct their own stand-up with their team members. A4 explained: "It was impossible for me to do that again with my team." 


\subsection{Lebanese Clients Vs Western Clients}

The mangers expressed the difference in working with different clients. They characterized them into two groups: the Western clients, North America and Western Europe, and Lebanese clients. Managers felt that it is easier to work with the Western clients in comparison to the Lebanese clients. V4 explained how transparency is key when dealing with the Western market: "Working with Western market, is easy. What you have to do is to be transparent and say things as they are. Working with the Lebanese market is much harder."

This is due to several factors pointed out by the mangers. First, the Western clients value time and provide a logical estimate for task completion. V2 explained: "In their business culture, they are committed to the set dates. Also, they are very reasonable in discussing the time required to complete each product." Lebanese clients tend to put unrealistic deadlines for their suppliers. Second, they are structured, well organized, and cooperative. V3 explained: "They did their due diligence by preparing the requirements. And they work with you in order to have their work done properly and with high quality. You will not find them as clients you'll find them as partners." Third, they have a collaborative nature and seek to have a successful product. V14 said: "If you have something a bottleneck in certain place and you give the good argument, they will understand and they will help you solve it." Fourth, Western clients are specific about their requirements. V4 explained: "They know exactly what you can do and what they have want. They know their budget and give you the righteous amount for your work."

On the contrary, dealing with Lebanese clients was harder and less efficient. Delays in project time affect the client, as well as the software development company that is keeping resources dedicated for a certain project longer than planned. The coming paragraphs explain several reasons why agile software development companies find difficulty in achieving results with Lebanese clients.

Lebanese clients show low levels of responsiveness to the requests from the software development company. This causes major delays in the due date. A3 explained: "The collection of data was the most difficult part." V3 expressed how clients delay in presenting the required information: "The client will delay in answering back our requirements to get their feedback. So this will lead us not to meet a deadline at a certain point." With clients responding poorly, gathering needed information was a challenging process. A1 explained a case where their client was a university in Lebanon and the project was delayed: "We knew that the educational system is slow by design but we weren't expecting 3 months of delay. Every time we requested data or a specification it would take days and weeks for them to respond." Practitioners indicate how they try to find innovative ways to keep the client engaged and motivate the client to respond promptly. A1 expressed: "We have to sometimes create ways to put pressure like sending emails, calling and texting in the same day."

Managers expressed how certain Lebanese clients stated their initial commitment to the development process, but through the development, the clients' enthusiasm decreased and their commitment became minimal. V2 claimed: "Lebanese client's availability and commitment of staff is not always as promised."

In addition, employees at the clients' premises may show resistance to change and feel threatened by it. V14 explained: "Some employees resist the change because our solutions are seen as a threat. You're taking my work they would say." 
Lebanese clients are not familiar with the software development industry thus they are threatened by it. So they tend to resist the suggested changes. According to V2: "Some will just try to punch holes in the solution being developed for them."

Furthermore, at the clients' side, employees require the presence and interference of the manager at every simple bottleneck. S6 explained a situation where a change in the colour used in the design was needed and the client executive requested a meeting with the manager regarding this issue.

\section{Discussion}

In this research we explore the conflicting trade-offs between adoption and tailoring of agile information systems development methods. On the one hand, agile methods originate in the Global North. Hence, agile methods represent a "northern" idea being propagated and adopted in the Global South. On the other hand, self-organising teams are empowering and emancipatory. Agile is about taking power away from managers and putting more responsibility into the hands of selforganising software development teams.

The research around agile tailoring is predicated on the assumption that methods must be adapted to the local context [5]. Conventionally, agile method tailoring tends to focus on factors such as business domain, technology stack and project characteristics (such as project scope or size and disposition of teams).

Our approach here, in contrast with conventional agile method tailoring, has been to address agile tailoring from an ICT4D perspective. So our perspective is not "how should Lebanese software development practices change in order to adopt agile methods" but rather "what adaptations to agile methods are required to achieve success with the approach in developing countries."

- RQ1: What can a critique, using post-colonial perspective, reveal about information system development methods?

Our study suggests that agile development needs to expand its corpus of basic frameworks. Agile tailoring should consider the cultural setting in which it is implemented. We suggest, a post-colonial reading of agile information systems development adds important dimensions to our understanding of agile tailoring. While one may argue that the differences between small and medium-sized software companies in the South are more significant than the difference between small software companies in the North and South, we advocate that the difference in agile methods adoption vary, using post-colonial theory, according to Global North and South perspective.

- RQ2: What challenges do we face in using a post-colonial perspective?

We found that post-colonial theory, and Southern theory more generally, is a substantial body of research. While the concepts around hybridity in post-colonial theory are helpful, we did not see many previous attempts to apply this to informations system management.

- RQ3: What can a post-colonial perspective reveal about adaptation to a specific context of information system development methods? 
We found that retrospectives, as described by the agile methodology, were rejected when introduced to practitioners in Lebanon. Culturally, practitioners are not comfortable with public self-evaluation or criticism. Thus, even though retrospective is a major agile ceremony, it is difficult to apply.

We know a lack of end-user involvement is challenging for agile organisations. Practitioners revealed how dealing with Lebanese clients is harder and less efficient than dealing with clients from the USA. Using post-colonial theory, we can say the Lebanese practitioners in our study successfully mimicked agile actions with "Western" clients. But the same mimicry action revealed potential negative impact on the relationship with the Lebanese clients who are not used to such involvement in the developing process.

Hence, we suggest the main contribution to knowledge arising from our research is that post-colonial theory offers new insights into agile method tailoring. Specifically, we propose that conceptions of tailoring needs to look beyond projectspecific characteristics and consider wider contexts.

We have enhanced the rigour of this research by collecting a substantial corpus of data and conducting detailed and thorough analysis. However, all interviewbased research suffers the drawback that is relies on self-reporting from respondents. We have used triangulation, of different roles and companies, to mitigate this. But we have been able to directly measure the impacts of agile methods on product quality or developer productivity.

\section{Conclusions}

In this research we have adopted a post-colonial stance, in order to understand the conflicting trade-offs between the adoption and tailoring of agile information system development methods. We concede that on the one hand, agile methods are a "northern" or "Western" innovation being copied and adopted around the world. And yet, on the other hand, agile methods undermine hierarchical management models and offer opportunities for empowerment within software development teams. Agile methods have been shown to have the potential for improved productivity and product quality.

To investigate these conflicting influences, we have undertaken a case study in three software development companies in four locations within Lebanon. Lebanon is emerging as a regional centre for outsourced software development in the middle east and gulf states. We conducted 31 semi-structured, open-ended, recorded and transcribed practitioner interviews.

The practitioners in our study struggled with the public self-evaluation mechanisms that agile method advocate for team learning. Also, we found local business clients were not well-informed about participation in agile projects. On the other hands, we found practitioners adept at using a rich variety of communication methods for overcoming geographical distance.

While agile tailoring is not a new concept, it has predominantly focused on project characteristics in the past. We think the post-colonial perspective presents a new and original framing on the tailoring concept by placing agile methods in a wider "Southern" context. 
We are currently investigating adaptation of selected agile ceremonies to better suite the Lebanese context. We will then observe the challenges faced and any benefits achieved.

\section{References}

1. Bass, J.M., Haxby, A.: Tailoring Product Ownership in Large-Scale Agile Projects: Managing Scale, Distance, and Governance. IEEE Software 36(2), 58-63 (Mar 2019)

2. Bass, J.M.: Artefacts and agile method tailoring in large-scale offshore software development programmes. Information and Software Technology 75, 1-16 (Jul 2016)

3. Bass, J.M.: Agile Software Development in Lebanon (Feb 2020). https://doi.org/10.17866/rd.salford.11887824.v1

4. Bhabha, H.K.: The Location of Culture. Routledge Classics, Abington, Oxon, 2 edn. (Sep 2004)

5. Campanelli, A.S., Parreiras, F.S.: Agile methods tailoring - A systematic literature review. Journal of Systems and Software 110, 85-100 (Dec 2015)

6. de Cesare, S., Patel, C., Iacovelli, N., Merico, A., Lycett, M.: Tailoring Software Development Methodologies in Practice: A Case Study. Journal of Computing and Information Technology 16(3), 157-168 (Oct 2004), number: 3

7. Comaroff, J., Comaroff, J.L.: Theory from the South. Routledge, Abingdon, Oxon (Jan 2012)

8. Conboy, K., Fitzgerald, B.: Method and Developer Characteristics for Effective Agile Method Tailoring: A Study of XP Expert Opinion. ACM Trans. Softw. Eng. Methodol. 20(1), 2:1-2:30 (Jul 2010)

9. Connell, R.: Using southern theory: Decolonizing social thought in theory, research and application. Planning Theory 13(2), 210-223 (May 2014)

10. Dingsøyr, T., Nerur, S., Balijepally, V., Moe, N.B.: A decade of agile methodologies: Towards explaining agile software development. Journal of Systems and Software 85(6), 1213-1221 (Jun 2012)

11. Foucault, M.: Archaeology of Knowledge. Routledge, London ; New York, 2 edition edn. (May 2002)

12. Frenkel, M., Shenhav, Y.: From Americanization to Colonization: The Diffusion of Productivity Models Revisited:. Organization Studies 24(9), 1537-1561 (Nov 2003)

13. Frenkel, M., Shenhav, Y.: From Binarism Back to Hybridity: A Postcolonial Reading of Management and Organization Studies. Organization Studies 27(6), 855-876 (Jun 2006)

14. Goss, J.: Postcolonialism: Subverting whose empire? Third World Quarterly 17(2), 239250 (Jun 1996)

15. Haxby, A., Lekhi, R.: Building Capacity in Kenya's ICT Market Using Cross-Border Scrum Teams. In: Information and Communication Technologies for Development. pp. 359-366. IFIP Advances in Information and Communication Technology, Springer, Cham (May 2017)

16. Hoda, R., Noble, J., Marshall, S.: Self-Organizing Roles on Agile Software Development Teams. IEEE Transactions on Software Engineering 39(3), 422-444 (2013)

17. International, Q.: NVivo qualitative data analysis software, https://www.qsrinternational.com/nvivo/home

18. Kreps, D., Bass, J.M.: Southern Theories in ICT4D. In: Nielsen, P., Kimaro, H.C. (eds.) Information and Communication Technologies for Development. Strengthening SouthernDriven Cooperation as a Catalyst for ICT4D. pp. 3-13. IFIP Advances in Information and Communication Technology, Springer International Publishing, Cham (2019)

19. Mir, R., Mir, A.: From the Colony to the Corporation: Studying Knowledge Transfer Across International Boundaries. Group \& Organization Management 34(1), 90-113 (Feb 2009)

20. Mohallel, A.A., Bass, J.M.: Agile Software Development Practices in Egypt SMEs: A Grounded Theory Investigation. In: Nielsen, P., Kimaro, H.C. (eds.) Information and Communication Technologies for Development. Strengthening Southern-Driven Cooperation as a Catalyst for ICT4D. pp. 355-365. Springer International Publishing, Cham (2019)

21. Noll, J., Razzak, M.A., Bass, J.M., Beecham, S.: A Study of the Scrum Master's Role. In: Product-Focused Software Process Improvement. pp. 307-323. Lecture Notes in Computer Science, Springer, Cham (Nov 2017) 
22. Ravishankar, M.N., Pan, S.L., Myers, M.D.: Information technology offshoring in India: a postcolonial perspective. European Journal of Information Systems 22(4), 387-402 (Jul 2013)

23. Regassa, Z., Bass, J.M., Midekso, D.: Agile Methods in Ethiopia: An Empirical Study. In: Information and Communication Technologies for Development. IFIP Advances in Information and Communication Technology, vol. 504, pp. 367-378. Springer, Cham, Yogyakarta, Indonesia (May 2017)

24. Said, E.W.: Orientalism. Penguin Books, London, 25th anniversary ed with 1995 afterword ed edition edn. (2003)

25. Sein, M.K., Thapa, D., Hatakka, M., Sæbø, y.: A holistic perspective on the theoretical foundations for ICT4D research. Information Technology for Development 25(1), 7-25 (Jan 2019)

26. Silvia Masiero: Subaltern studies: Advancing critical theory in ICT4D. In: Twenty-Sixth European Conference on Information Systems (ECIS 2018). vol. 162, pp. 1 - 13. Association for Information Systems, Portsmouth, UK (2018)

27. Spivak, G.C.: Can the subaltern speak? In: Nelson, C., Grossberg, L. (eds.) Marxism and the Interpretation of Culture. University of Illinois Press, Urbana, reprint edition edn. (Oct 1987)

28. Spivak, Gayatri Chakravorty: A Critique of Postcolonial Reason: Toward a History of the Vanishing Present. Harvard University Press (May 1999)

29. Stray, V., Sjøberg, D.I.K., Dybå, T.: The daily stand-up meeting: A grounded theory study. Journal of Systems and Software 114, 101-124 (Apr 2016)

30. Walsham, G.: Doing interpretive research. European Journal of Information Systems 15(3), 320-330 (Jun 2006) 